achieved in their respective thin-film structures, opening the door to in situ cooling of semiconductors. As reported in the July 14 issue of Applied Physics Letters, the researchers used low-pressure metalorganic chemical vapor deposition to grow 100-period Si-doped superlattices with 10-nm AlGaAs barriers and 10-nm AlGaAs wells. The group chose the AlGaAs system for its compatibility with GaAs, and the particular low-Al barrier and well phases, because of their low thermal conductivity compared to GaAs, while exhibiting comparable electrical conductivity. In addition, they can be doped to a very high level.

The scientists fabricated stand-alone microcoolers using standard lithography techniques and $\mathrm{Ni} / \mathrm{Au} / \mathrm{Ge} / \mathrm{Au}$ as wire bonding contacts, and they mounted the device on a copper heatsink for analysis. The specific series resistance including contact and superlattice resistance was determined to be $3.2 \times 10^{-6} \Omega \mathrm{cm}^{2}$. In the measured temperature range of $25-100^{\circ} \mathrm{C}$, the cooling effect increased from $0.8^{\circ} \mathrm{C}$ to $2^{\circ} \mathrm{C}$, indicating increasing efficiency with higher temperatures, according to the researchers. Size reduction from $120 \mu \mathrm{m}^{2} \times 120 \mu^{2}$ to $60 \mu^{2} \times 60 \mu \mathrm{m}^{2}$ increased the cooling effect from $0.67^{\circ} \mathrm{C}$ to $0.8^{\circ} \mathrm{C}$, but the trend did not continue with further size reduction. The researchers expect significant improvements in cooling performance through optimization of contact resistance doping level, superstructure, and geometric parameter adjustment.

Alfred A. ZINN

\section{Nanocomposites Made from Renewable Resources Exhibit Good Mechanical Properties}

Interest in preparing polymeric materials from renewable, plant-based resources has grown in recent years. Plant oils are particularly attractive raw materials because of their worldwide abundance, and "green" polymers are already being used in inks and coatings. However, these materials do not offer the mechanical properties necessary for structural applications. A group of researchers at Kyoto University and Toyota Central R\&D Labs has now used plant oils and clay to prepare nanocomposites that show strength, flexibility, and biodegradability. They report their results in the July 1 issue of Chemistry of Materials.

The team prepared the nanocomposites by cross-linking either epoxidized soybean oil (ESO) or epoxidized linseed oil (ELO) in the presence of montmorillonite clay modified with organic groups. Wide-angle $\mathrm{x}$-ray diffraction and transmission electron microscopy were used to characterize the insoluble polymeric network. Dynamic viscoelasticity measurements showed that the storage moduli of the nanocomposites increased as a function of clay content. In the absence of clay, only a very soft film was obtained, while nanocomposites with $5-15 \%$ clay content displayed flexibility and good mechanical strength. The storage modulus of the ELO clay nanocomposite was greater than that for ESO clay, probably due to a higher cross-linking density in the ELO polymer, according to the researchers.

CATHERINe Oertel

\section{$\mathrm{Si} / \mathrm{TiB}_{2}$ Microcomposite as Li-Ion Battery Anode Material Yields High and Stable Discharge Capacity}

Graphite is currently the main anode material for lithium-ion batteries, the power source of choice for portable electronics such as cellular phones, laptop computers, and video cameras. However, the performance of graphite anodes suffers from a relatively low theoretical maximum capacity of $372 \mathrm{mAh} / \mathrm{g}$ and irreversible capacity loss in the first cycle. P.N. Kumta, I.-S. Kim, and G.E. Blomgren of Carnegie Mellon University have found that the use of a microcomposite material consisting of $\mathrm{TiB}_{2}$ as an inactive host matrix and $\mathrm{Si}$ as the finely dispersed active phase minimizes the detrimental stresses during the charge/discharge

\section{Fast And Contact-Free}

The LSM 5 Pascal Laser Scanning Microscope efficiently analyzes microstructures.

Precision optics, innovative

scanning strategies \& powerful, modular software packages guarantee maximum operating convenience and flexibility. Scanning field can be expanded by 3D stitching.

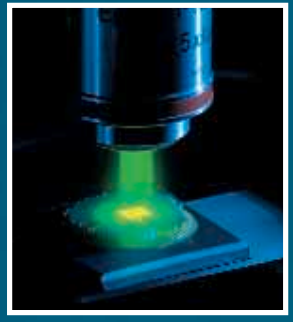

For more information, call 800-233-2343

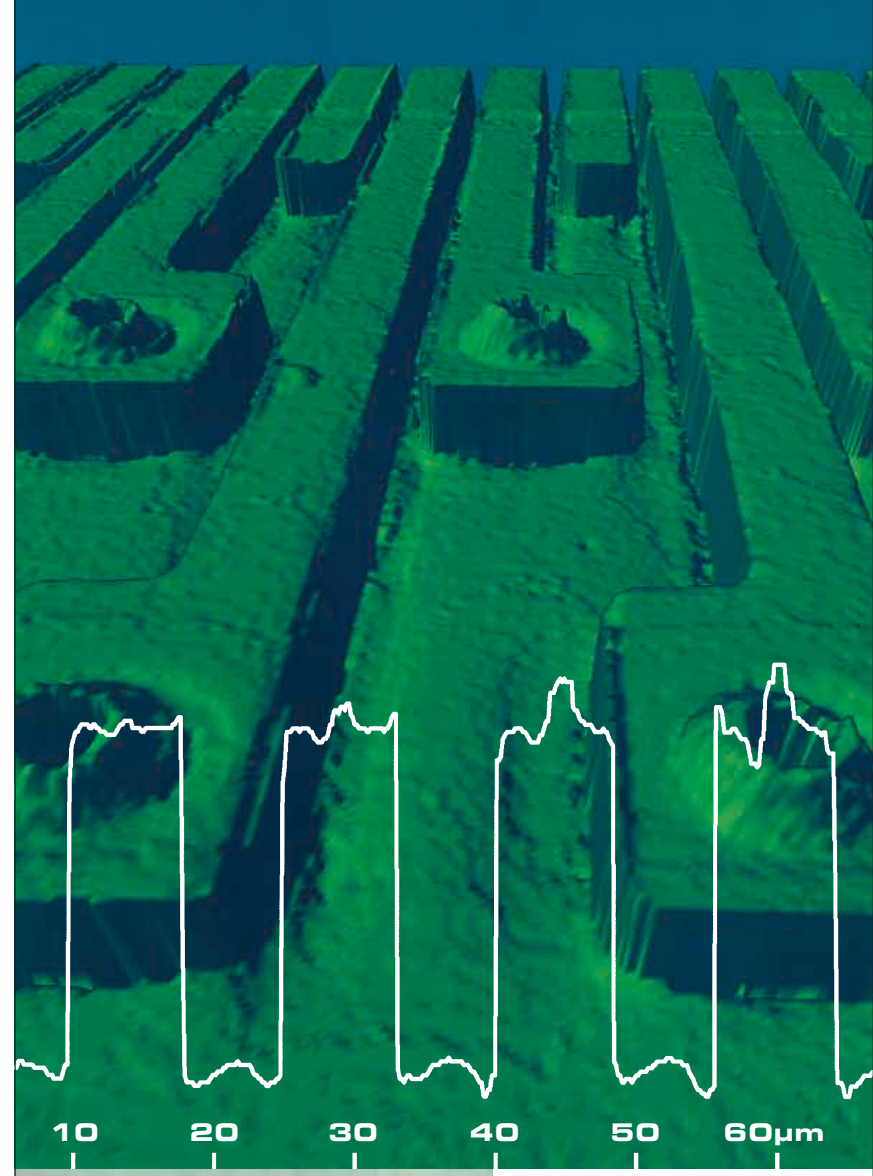

Carl Zeiss Microlmaging, Inc Thornwood, NY 10594 micro@zeiss.com zeiss.com/lsm-mat

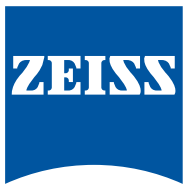

We make it visible.

For more information, see http://advertisers.mrs.org 